\title{
SHIFTING TO TRIPLE VALUE HEALTHCARE: REFLECTIONS FROM ENGLAND
}

\author{
Anant Jani, Sven Jungmann, Muir Gray* \\ * correspondences should be addressed to muir.gray@medknox.net
}

\begin{abstract}
Increasing need and demand because of growing and aging populations combined with stagnant or decreasing resources being invested into healthcare globally mean that a radical shift is needed to ensure that healthcare systems can meet current and future challenges. Quality-, safety- and efficiency-improvement approaches have been used as means to address many problems in healthcare and while they are essential and necessary, they are not sufficient to meet our current challenges. To build resilient and sustainable healthcare systems, we need a shift to focus on triple value healthcare, which will help healthcare professionals improve outcomes at the process, patient and population levels while also optimising resource utilisation. Here we present a brief history of the Quality and Evidencebased Healthcare model and then describe how value emerged as a predominant theme in England. We then highlight the four solutions that we, as part of the RightCare programme, designed and refined in the English NHS to turn theory into practice:

- Triple Value Healthcare

- Culture Change, Accountability and Stewardship

- Systems, Networks and Pathways

- Using knowledge and technology as enablers
\end{abstract}

We end with a description of how triple value is being introduced into Germany and steps that can be taken to facilitate its adoption.

Keywords: Value based healthcare; value; culture change; outcomes; stewardship 


\section{Introduction: Why value?}

Many countries have decided to stop the growth of investment in healthcare or at least slow the growth despite need and demand continuing to increase. These decisions were exacerbated by the 2008 global financial collapse, though Germany has been addressing these issues even before that - take, for example, the Rürup Commission's goal of reducing healthcare expenditure by billions of euros in 2003. ${ }^{1}$ In England, the 2008 global financial collapse drove the then Chief Executive of the English National Health Service (NHS), David Nicholson, to issue the Nicholson Challenge which called for $f 20$ billion in savings by 2014$2015 .^{2}$

Setting aside the financial challenges, even if money were not a problem, growing concern about overdiagnosis and overtreatment is also raising the issue of value. The Choosing Wisely campaign ${ }^{3}$ and the BMJ's campaign on Too Much Medicine and Over Diagnosis ${ }^{4}$ highlight these issues and raise the importance of thinking of value as well as evidence based decision making and quality.

In response to these challenges, one of the authors, Sir Muir Gray, co-established the NHS England RightCare programme in 2010, which aimed ${ }^{2,5}$ :

To increase value and address unwarranted variations in spend, activity and outcomes through: using programme budgeting and marginal analysis to improve clinical commissioning; better value commissioning with knowledge, information and coaching; reducing the use of lower value interventions; reducing unwarranted variation in referral rates to elective care; and improving patient satisfaction and reducing the costs of treatment, by involving patients in shared decision-making.

RightCare represented a shift in the care delivery models used previously, as highlighted in the table below:

\begin{tabular}{|l|l|}
\hline Quality \& Evidence-based Model & Triple Value Healthcare Model \\
\hline $\begin{array}{l}\text { The Aim is on effectiveness, quality } \\
\text { and safety outcomes }\end{array}$ & $\begin{array}{r}\text { The Aim is triple value \& greater equity } \\
\text { Allocative value, determined by how the } \\
\text { assets are distributed to different sub groups } \\
\text { in the population }\end{array}$ \\
& $\begin{array}{l}\text { Technical value, determined by how well } \\
\text { resources are used for all the people in need } \\
\text { in the population }\end{array}$ \\
& $\begin{array}{l}\text { Personalised value, determined by how well } \\
\text { the decisions relate to the values of each } \\
\text { individual }\end{array}$ \\
\hline Good service for known patients & $\begin{array}{l}\text { Personalised service for all the people affected in } \\
\text { the population }\end{array}$ \\
\hline Improvement through competition & $\begin{array}{l}\text { Improvement through collaborative systems and } \\
\text { networks with patients \& carers as equal partners }\end{array}$ \\
\hline Transformation attempted by & Transformation by culture change \& digital \\
\hline
\end{tabular}


reorganisation \& more money

Clinicians act as the users of their

institution's resources

\section{knowledge services}

Clinicians feel they are the stewards of the

population's resources

The Triple Value Healthcare model has been in use in England since 2010 via the English NHS' RightCare programme, the longest running and most deeply embedded national programme focused on value based healthcare that the authors are aware of, and this new model is also being used in Wales (Prudent Healthcare ${ }^{6}$ ), Scotland (Realistic medicine ${ }^{7}$ ) and Saudi Arabia (Model of Care programme).

In this manuscript, we briefly highlight the history of the Quality and Evidence-based Healthcare model and then describe how value emerged as a predominant theme in England. We then highlight the four solutions that we, as part of the RightCare programme, designed and refined in the English NHS to turn theory into practice:

- $\quad$ Triple Value Healthcare

- Culture Change, Accountability and Stewardship

- Systems, Networks and Pathways

- Using knowledge and technology as enablers

We end with a description of how triple value is being introduced into Germany and steps that can be taken to facilitate its adoption.

\section{Quality and Evidence-based Healthcare}

The Zeitschrift für Evidenz, Fortbildung und Qualität epitomises the revolution not only of medical journals, but of professions in the last century. Journals started as places for recording scientific research and then they saw their task as contributing to continuing medical education producing material that physicians could use to maintain their knowledge and skills and improve them.

The introduction of quality in healthcare and the development of a new model of care in which doctors had to work within certain constraints and guidelines is fifty years old and this year marks the fiftieth anniversary of Avedis Donabedian's classic article in the Millbank Quarterly, ${ }^{8}$ generally regarded as the article that launched quality assurance. In many countries, however, little was done until a number of reports were produced that emphasised the importance of quality of healthcare. For example, the Institute of Medicine in Washington came two reports, one called Causing the Quality Chasm ${ }^{9}$ and the second, focusing primarily on safety, To Err Is Human ${ }^{10}$. In the United Kingdom, the stimulus came from a report called An organisation with a memory ${ }^{11}$ emphasising the need for organisations to become learning organisations.

The development of evidence based decision making is also about twenty years old. This origin can be traced to the development of the McMaster Medical School and the 
publication in 1986 of the landmark text called Clinical Epidemiology, subtitled $A$ basic science for clinical practice ${ }^{12}$.

In the 1990's the McMaster Faculty decided that although they had got clinical epidemiology on the curriculum it still sounded too academic, and they developed a style of clinical practice that they called evidence based medicine, which is defined below:

"Evidence based medicine is the conscientious, explicit, and judicious use of current best evidence in making decisions about the care of individual patients. The practice of evidence based medicine means integrating individual clinical expertise with the best available external clinical evidence from systematic research. By individual clinical expertise we mean the proficiency and judgment that individual clinicians acquire through clinical experience and clinical practice. Increased expertise is reflected in many ways, but especially in more effective and efficient diagnosis and in the more thoughtful identification and compassionate use of individual patient's predicaments, rights, and preferences in making clinical decisions about their care." ${ }^{13}$

Evidence based medicine achieved a high profile in the 1990's partly due to the work of the Faculty of McMaster University but also, certainly in Europe, due to the decision by David Sackett to accept the Chair at the University of Oxford to set up the Centre for Evidence Based Medicine.

\section{The emergence of value}

Avedis Donabedian also played a key part in developing the thinking around value in healthcare. In his classic three volumes on quality published in $1980^{14}$ he included a graph depicting the relationship between the investment of resources, benefit and harm.

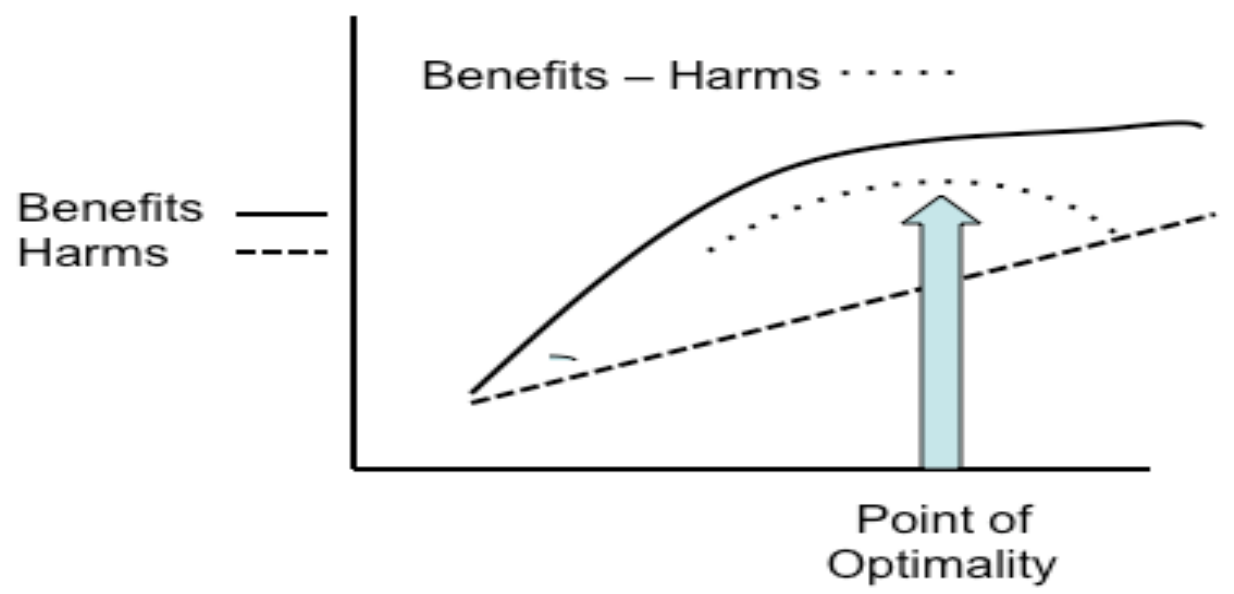


Improving quality changes the relative position of the two lines for benefit and harm but does not change the nature of the relationship.

What he pointed out was that it was possible to go beyond the point of optimality, defined by Donabedian as "the most advantageous balancing of costs and benefits" 15 , where the costs outweighed the benefits. In discussion with clinicians and the public, a number of topics emerged in which they feel that the service had gone beyond the point of optimality. Common topics raised in meetings include:

- Antibiotic prescription

- Polypharmacy in people with multiple morbidity

- Very elderly people with multiple morbidity dying in intensive care

- Elective surgery

- Recent increases in imaging and diagnostic use

\section{Triple Value Healthcare}

The early thinking on value-based healthcare was led by Professor Michael Porter from Harvard Business School. His contributions have been very important in bringing the concepts of value-based healthcare to healthcare professionals and when we began to implement his work in England via the RightCare programme we found that it did not meet all of our needs. One of the key limitations in Porter's approach is that it is not designed for universal healthcare systems, which have two key constraints: 1 . an explicit and legally required commitment to cover the needs of the whole population and 2 . to meet these needs within a finite budget.

As a starting point in England's value journey, we realised the importance of creating a common language around value in universal healthcare systems so that there was no confusion or ambiguity among stakeholders, including patients, about what value meant and why it was important. The framework below addresses value at three different levels ${ }^{16}$

\begin{tabular}{|c|c|}
\hline Personal Value & $\begin{array}{l}\text { Value at the level of the Patient } \\
\text { - Is the care that you are delivering meeting the needs and } \\
\text { expectations of the patients to whom you are accountable? } \\
\text { - The needs and expectations will include objective clinical } \\
\text { outcomes (e.g. lower blood pressures) as well as subjective } \\
\text { outcomes (e.g. being able to golf or play with one's } \\
\text { grandchildren) }\end{array}$ \\
\hline Technical Value & $\begin{array}{l}\text { Value at the level of the Intervention } \\
\text { - What are the outcomes (patient, population and process)) } \\
\text { delivered by an intervention and what are the resources } \\
\text { (money, time, space, carbon) needed to deliver those } \\
\text { outcomes? } \\
\text { - In addition to looking at the absolute value of an }\end{array}$ \\
\hline
\end{tabular}




\begin{tabular}{|l|l|}
\hline Allocative Value & $\begin{array}{c}\text { intervention, we must also consider the relative value, which } \\
\text { will help in the identification and utilization of higher value } \\
\text { interventions as well as disinvestment from lower value } \\
\text { interventions }\end{array}$ \\
\hline $\begin{array}{l}\text { Value at the level of the Population } \\
\text { (money, time, space, carbon) to meet the needs of the entire } \\
\text { (mopulation to whom you are accountable? } \\
\text { ponsideration also needs to be made about how to balance } \\
\text { the individual (e.g. personal value) and population needs - } \\
\text { everyone will not be able to get everything they want and an } \\
\text { open and honest dialogue with the public is essential here. }\end{array}$ \\
\hline
\end{tabular}

\section{Culture Change, Accountability \& Stewardship}

"Culture refers to what stands behind and guides behaviour rather than the behaviour as such."17

Changing and building a culture is not easy but it is essential if we hope to shift to triple value based healthcare. In our work with RightCare we found that culture was the first place we needed to start and changing culture was driven first by knowledge of the basic concepts of value based healthcare and next by giving healthcare professionals a means by which they could realise and hold themselves accountable for value based healthcare principles.

For the accountability question, the two key questions healthcare professionals have to ask themselves are:

1. Who am I holding myself accountable to?

2. What am I holding myself accountable for?

\section{Who are you holding yourself accountable to?}

For most healthcare professionals there are a variety of entities they can hold themselves accountable to: themselves, their employer, their profession, their patients and/or the population they serve. It is a difficult task to create a balance between establishing accountability across these entities, but it is essential that a balance be created so the needs and expectations of all of these entities are met. When designing this accountability balance for yourself, it is also good to remember that most healthcare professionals pursued their profession to deliver the best outcomes to the patients and populations they serve and care for. 
From a value perspective, there are two key areas healthcare professionals need to hold themselves accountable for: outcomes and resource utilisation.

There are three main categories of outcomes in healthcare: process, patient and population outcomes. Most data collected in healthcare focus on process outcomes, which is certainly necessary but it is important to remember that process outcomes are usually dependent on the specific healthcare service structures (networks and pathways used) which may change over time and having an ideal set of processes is no guarantee that patients and populations are actually benefiting or are in good health. As a healthcare community, we need to make a commitment to additionally focus more on patient and population level outcomes.

Resources utilised in healthcare certainly include money, but in addition to this, we must also consider time (that of healthcare professionals as well as patients), use of space and use of carbon.

The matrix below highlights accountability for outcomes and resource stewardship across the process, patient and population domains.

\begin{tabular}{|l|l|l|l|}
\hline & Processes & Patients & Populations \\
\hline Outcomes & $\begin{array}{l}\text { Ensuring that patients } \\
\text { and populations get } \\
\text { evidence-based } \\
\text { interventions that } \\
\text { adhere to validated } \\
\text { guidelines and } \\
\text { protocols }\end{array}$ & $\begin{array}{l}\text { Ensuring that the care } \\
\text { provided to patients } \\
\text { focuses on the outcomes } \\
\text { (both clinical and } \\
\text { personal) that matter } \\
\text { most to patients }\end{array}$ & $\begin{array}{l}\text { Ensuring that all people in } \\
\text { need receive care }\end{array}$ \\
\hline $\begin{array}{l}\text { Resource } \\
\text { Stewardship }\end{array}$ & $\begin{array}{l}\text { Ensuring that resources } \\
\text { are utilized efficiently } \\
\text { and effectively to } \\
\text { minimize waste }\end{array}$ & $\begin{array}{l}\text { Ensuring that patients do } \\
\text { not get unnecessary } \\
\text { interventions }\end{array}$ & $\begin{array}{l}\text { Ensuring that the healthcare } \\
\text { resources available (money, } \\
\text { time, expertise) are utilized } \\
\text { for the maximum benefit of } \\
\text { the population being served }\end{array}$ \\
\hline
\end{tabular}

Creating the right balance across these areas takes time and requires multi-stakeholder engagement to ensure that multiple perspectives are being taken into account when determining what the measure and, thus, hold oneself accountable for. An example output from this type of process can be seen from some of the work we have done with colleagues in Connecticut, US for early intervention in psychosis services. ${ }^{18}$

\section{Systems, Networks and Pathways}

Having a common understanding of value based healthcare as it applies to universal healthcare systems, we next needed to move onto implementation and identifying the ideal place for delivering triple value. 
Healthcare generally operates on three different levels, Systems, Networks and Pathways ${ }^{19}$ :

Systems: The system defines the outcomes (which are health equity based and service independent) delivered to patients

Networks: The network are comprised of the individuals and organisations who deliver the outcomes (i.e. GPs, surgeons, social care, etc.)

Pathways: The pathways are defined by how the outcomes are delivered.

Healthcare Services: The Network and Pathway structures used to deliver outcomes to patients and populations.

Value can be delivered at all of these levels but qualifying the above elements further, one sees that the healthcare system is the ideal level to deliver triple value. One can think of a system as being something that would be equally relevant across time and space, while the networks and pathways, which constitute healthcare services, will always be variable over time and space. Elaborating on this point, a system focuses on the outcomes that you want to deliver to patients and populations and this inherent focus on clinical needs means that a system will always be relevant because, for example, people with asthma existed 50 years ago and they will exist 50 years from now and there will be people with asthma in Berlin, Munich, Toronto, London, Durban, Delhi, Syndey, etc. The outcomes that we as healthcare professionals want to deliver to them will largely be the same - e.g. preventing asthma attacks, enabling people with asthma to live their life to the fullest, etc. The people who deliver the care (i.e. the networks) and the means they use to deliver the care (i.e. the pathways) will vary over time as our understanding of the disease increases and new technologies facilitate the delivery of better outcomes and as political mandates change the structure of the healthcare service. Similarly, the networks and pathways will also vary across geographies because of differences in resource availability, local demographic considerations and local politics. Across all of this, however, the ideal is that we adhere to considerations of health equity to ensure that a patient with a given condition should expect and receive the same outcomes irrespective of when or where they live.

\section{Using knowledge \& Technology as enablers}

Knowledge and technology are two key enablers that we used to enable healthcare professionals and systems to deliver triple value healthcare.

\section{Knowledge}

Knowledge is the enemy of disease, the application of what we know will have a bigger impact than any drug or technology likely to be introduced in the next decade. 
To deliver value, we must

strategically and effectively

use knowledge from

multiple sources and from

different areas of the

healthcare system.

Knowledge can generally

be derived from

experience, which is

normally captured in the

form of case studies and

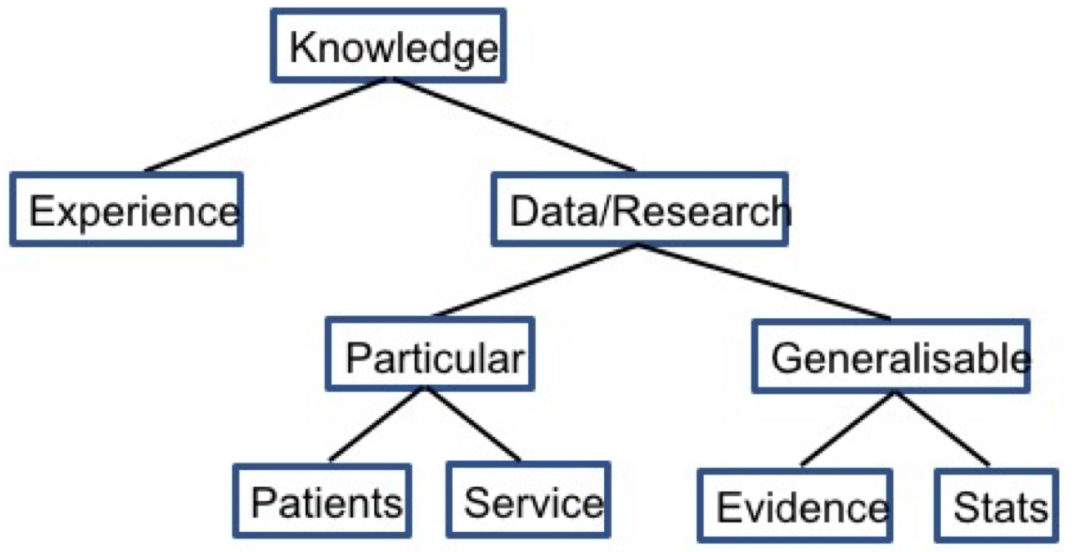

data/research. Knowledge

from data/research can be further subdivided into particular and generalizable knowledge.

This is demonstrated in the adjacent diagram.

In addition to the types of knowledge, we also need to be aware of the places the knowledge comes from. There are generally three elements used to build all healthcare systems:

- Structural elements (e.g. where care is delivered - hospitals, primary care practices, community clinics, etc.)

- Finance elements (e.g. tax-based, insurance-based, social welfare, out of pocket, etc.)

- Patients and their clinical conditions (e.g. patients with clinically-defined conditions like asthma or COPD; patients with symptoms like breathlessness; patients that belong to a group like frail elderly)

These elements will further aggregate into different levels (systems, networks and pathways, described above) depending on the local resources available and the demographics of the population to which care is delivered.

Knowledge across these areas and domains can be used by healthcare professionals to make strategic, operational and tactical decisions, as well as judgements, about the design and delivery of healthcare services to ensure they are delivering the best patient and population outcomes while also optimising resource utilisation - i.e better value healthcare.

\section{Technology}

Rapidly growing scientific output ${ }^{20,}{ }^{21}$ has made it difficult to appraise new publications and to translate knowledge into practice efficiently ${ }^{9,22}$. Medicine's body of knowledge has moved from something like a desert to something comparable to a jungle. While today's clinicians still base many decisions on incomplete evidence, they now also need to tackle an abundance of information that has become unmanageable. ${ }^{18}$

Information and Computing Technologies (ICT) serve as a critical enabler that can help distil the right insights from the noisy data backdrop and adapt to individual patients' and clinicians' needs, e.g. through machine learning technologies, which could ultimately lead to 
the emergence of Learning Healthcare Systems. ${ }^{23}$ The Institute of Medicine defines these as systems in which "science, informatics, incentives, and culture are aligned for continuous improvement and innovation, with best practices seamlessly embedded in the delivery process and new knowledge captured as an integral by-product of the delivery experience." 24

ICT can make it affordable to measure value-based outcomes (using instruments like ICHOM) by automating data collection and evaluation processes that thus far exceeded our capacities. Furthermore, digital technologies, particularly mobile health and ambient computing, can expand the continuum of care (including prevention) well into the everyday lives of citizens helping them manage their health independently of professional and informal care givers, which will be empowering and will also reduce the burden of care. Additionally, ICT-enabled networks can facilitate global interaction between and among patients, healthcare providers, and researchers, enhancing transparency, knowledge sharing, and serendipitous discoveries (e.g. when individuals with rare diseases connect).

These uses all fall under the umbrella term "eHealth", which has many definitions. The one that, in our view, is the most comprehensive definition is proposed by Eisenbach:

"E-health is an emerging field in the intersection of medical informatics, public health and business, referring to health services and information delivered or enhanced through the Internet and related technologies. In a broader sense, the term characterizes not only a technical development, but also a state-of-mind, a way of thinking, an attitude, and a commitment for networked, global thinking, to improve health care locally, regionally, and worldwide by using information and communication technology." 25

The ultimate aspiration of the term "eHealth" is to render itself obsolete, as it will increasingly become an integral part of healthcare.

Technology, however, also bears risks. It can harm social value by widening social divides, for example when digital literacy, personal wealth, or good digital infrastructures are required to access these emerging technologies. Arguably, those who stand to gain the most from these technologies might also be at highest risk of not being able to access them. This can only be avoided if innovators, healthcare professionals, and policy makers join forces to make novel technologies as accessible as possible. Innovators must cultivate a user centric design that is not just focused on patients, but also their professional and informal care givers. Healthcare providers must learn how to guide their patients on the use of modern technologies just like they advise them on medicines. Policy makers need to ensure that public education and infrastructure makes eHealth access possible independent of geography and personal finances. If we are able to strike this critical balance, technology in the form of eHealth and ICT will be an extremely powerful enabler for delivering triple value healthcare.

Within the RightCare programme, we harvested and disseminated knowledge in multiple ways $^{6}$ : 
- Atlases of Variation (both print and digital), which highlighted unwarranted variation in spend and outcomes

- Commissioning for Value Packs (both print and digital) which benchmarked payers across the country on spend and outcome

- Casebooks which highlighted best practice

These knowledge resources were used, and are currently being used, by RightCare as critical enablers to motivate and empower healthcare professionals to drive change as well as to innovate.

\section{Shifting to Triple Value Healthcare}

Shifting healthcare systems to triple value healthcare is not easy and it is not quick. In England, our journey started in 2010 and we will likely need several more years before triple value healthcare is as deeply embedded in the psyche of healthcare professionals as quality and evidence-based healthcare are. The key lessons we have learned from our journey are the importance of creating a common language from the start and engaging with all stakeholders from the start in the journey so that the culture can begin to change.

In Germany, this process has already started with the first value based congress, Deutscher Kongress Value Based Healthcare, organised in May 2016 by the Ärtzekammer Berlin, the British Medical Journal and the Value Based Healthcare programme at the University of Oxford $^{26}$. The Congress covered a variety of topics that addressed triple value healthcare at the local and national levels and had speakers and attendees from all over Germany. The discussions at the Congress were very encouraging and showed a real appetite for adopting triple value healthcare in Germany. Moving forward, we hope to creating a learning and sharing network with our colleagues in Germany and internationally so that we can continue to increase the triple value of healthcare systems globally.

\section{References}

1. Aris, B. (2003) Commission recommends reform of German health care. The Lancet. 362 : 1388.

2. National Audit Office. 2011. Delivering efficiency savings in the NHS. London: National Audit Office. https://www.nao.org.uk/wpcontent/uploads/2011/12/NAO_briefing_Delivering_efficiency_savings_NHS.pdf.

3. Choosing Wisely Campaign [Internet]. c2017 [cited 2017 Aug 2]. Available from http://www.choosingwisely.org/.

4. Too Much Medicine [Internet]. c2017 [cited 2017 Aug 2]. Available from http://www.bmj.com/too-much-medicine.

5. NHS England RightCare Programme [Internet]. c2017 [cited 2017 Aug 2]. Available from https://www.england.nhs.uk/rightcare/.

6. NHS Wales Prudent Healthcare [Internet]. c2017 [cited 2017 Aug 2]. Available from http://www.prudenthealthcare.org.uk/ 
7. The Scottish Government. 2017. Chief Medical Officer's Annual Report 2015-16: Realising Realistic Medicine. Scotland: NHS Scotland.

8. Donabedian A. (2005) Evaluating the quality of medical care. Milbank Memorial Fund Q. 1966;44(suppl):166-206. Reprinted in Milbank Q. 83:691-729.

9. Institute of Medicine. 2001. Crossing the Quality Chasm: A New Health System for the 21st Century. Washington, DC: The National Academies Press. https://doi.org/10.17226/10027.

10. Institute of Medicine. 2000. To Err Is Human: Building a Safer Health System. Washington, DC: The National Academies Press. https://doi.org/10.17226/9728.

11. England Department of Health. 2000. An Organisation with a Memory. London: The Stationary Office.

http://webarchive.nationalarchives.gov.uk/20130105144251/http://www.dh.gov.uk/prod_c onsum_dh/groups/dh_digitalassets/@dh/@en/documents/digitalasset/dh_4065086.pdf

12. Sackett D, Haynes RB, Tugwell P and Guyatt G. Clinical Epidemiology: A Basic Science for Clinical Medicine. Boston: Little Brown and Co, 1986.

13. Sackett DL, Rosenberg WMC, Gray JAM, Haynes RB, Richardson WS. (1996) Evidence based medicine: what it is and what it isn't. BMJ 312:71.

14. Donabedian A. The Definition of Quality and Approaches to Its Assessment. Vol 1-3. Michigan: Health Administration Press, 1980.

15. Donabedian A. (1990) The seven pillars of quality. Arch Pathol Lab Med 114: 1115-8.

16. Value Based Healthcare Programme [Internet]. c2017 [cited 2017 Aug 2]. Availabe from https://www.phc.ox.ac.uk/research/value-based-healthcare.

17. Alvesson, M. and Sveningsson, S. (2008) Changing Organizational Culture. Cultural change work in progress. Routledge. (p.36).

18. Srihari VH, Jani A, Gray M. (2016) Early Intervention for Psychotic Disorders: Building Population Health Systems. JAMA Psychiatry 73:101-2.

19. Jani A and Gray M. (2015) Outcomes as a foundation for designing and building population healthcare systems in England. BMJ Outcomes Article Collection p16. http://15762presscdn-0-11.pagely.netdna-cdn.com/wp-content/uploads/2016/08/BMJ-OutcomesArticle-Collection.pdf

20. Sadeghi M. (2017) Are Scholarly Journals in Search for Top Quality Articles or Authors in Search for High Quality Journals? J Reprod Infertil 18: 151-152.

21. "Global scientific output doubles every nine years" Nature Blogs, 7 May 2014, http://blogs.nature.com/news/2014/05/global-scientific-output-doubles-every-nineyears.html.

22. Niven DJ, Rubenfeld GD, Kramer AA, Stelfox HT. (2015) Effect of published scientific evidence on glycemic control in adult intensive care units. JAMA Intern Med 17: 801-9.

23. Foley T and Fairmichael F. 2015. The Potential of Learning Healthcare Systems. The Learning Healthcare Project. http://www.learninghealthcareproject.org/LHS_Report_2015.pdf

24. Institute of, M. e Learning Health System Series. 2015 [cited 20175 August]; Available from: http://www.nap.edu/catalog/13301/the-learning-health-system-series.

25. Eysenbach G (2001) What is e-health? J Med Internet Res 3: e20.

26. Deutscher Kongress Value Based Healthcare [Internet] c2017. [cited 2017 October 14] Available from https://www.aerztekammer-berlin.de/10arzt/26_Kongresse/09_ValueBased-Health-Care/index.shtml 